\title{
FOCO EPILEPTÓGENO PARASSAGITAL COM REVERSÃO DE FASE NO VERTEX: CORRELAÇÕES CLÍNICO-ELETRENCEFALOGRÁFICAS
}

\author{
DÉcIO MEGA* \\ MICHEL P. LISON **
}

Descargas focais primárias, originadas em lesões epileptógenas parassagitais, são dificilmente registradas no eletrencefalograma 17, 19. Por outro lado tais lesões podem determinar distúrbios eletrográficos à distância, dificultando, ainda mais, a interpretação do traçado. Esse conceito, desenvolvido por Jasper e col. (Penfield e Jasper ${ }^{15}$, Jasper ${ }^{6}$ e Tükel e Jasper ${ }^{19}$ ) ao descreverem o "sincronismo bilateral secundário" foi confirmado, posteriormente, por Ralston ${ }^{18}$, em bases experimentais.

Do ponto de vista clínico, tem sido referida a ocorrência de crises adversivas ${ }^{1,17,19}$ e crises de ausência ${ }^{10,14,17,19}$ em pacientes com foco epileptógeno na superfície mesial. Entretanto, com referência às crises acinéticas, não tem sido estabelecida relação idêntica, embora haja evidência de que a estimulação do giro cingulado, em animais, pode desencadear perda súbita do tono muscular, muito semelhante àquela verificada em pacientes com crises acinéticas ${ }^{10}$.

No presente trabalho estudamos a correlação eletroclínica em pacientes com foco epileptógeno parassagital, dandu ênfase à ocorrência de crises acinéticas.

\section{MATERIAL E METODOS}

Nosso material consta de 22 pacientes selecionados entre os 5.000 registrados em nosso serviço de eletrencefalografia nos últimos 5 anos. $O$ critério de seleção adotado foi puramente eletrencefalográfico: descargas focais projetando-se ao nivel da regiāo parassagital com reversão de fase no vértex.

Os registros eletrencefalográficos (EEG) foram obtidos com aparêlho Grass de 8 canais e os eletrodos colocados segundo a técnica internacional ${ }^{7}$. Analisamos os traçados obtidos em viǵlia, durante o sono e no decorrer da hiperpnéia. Deixamos de considerar os registros obtidos durante estimulação auditiva, luminosa, tactil ou nociceptiva. Procuramos analisar as descargas quanto à sua forma, voltagem e distribuição, excluindo-se do trabalho, aquelas que pudessem ser confundidas com espiculas normais do vértex. Foram igualmente excluidas as descargas fronto-centro-parietais que não apresentavam reversão de fase na linha média.

Departamento de Neurologia da Faculdade de Medicina de Ribeirão Prêto da Universidade de São Paulo (Prof. J. Armbrust Figueiredo): * Instrutor; ** Proressor Assistente. 


\title{
RESULTADOS
}

\author{
Analisamos nossos resultados sob os pontos de vista clínico e eletrence- \\ talográfico.
}

A idade dos pacientes variou de 2 meses a 14 anos, com acentuada predomináncia no grupo etário de 2 a 7 anos (82\%). Em 3 casos foi superior a 7 anos e em um, inferior a 2 anos (quadro 1). Quanto ao sexo 10 eram masculinos e 12 femininos.

Dos 22 pacientes estudados, $17(77 \%)$ apresentavam crises epilépticas, cujos tipos clinicos estāo representados no quadro 1 . Os 5 pacientes restantes nunca apresentaram qualquer manifestação convulsiva. As crises adversivas (10 casos) e as crises acinéticas (4 casos) predominaram sôbre as demaís. O exame neurológico evidenciou retardo do desenvolvimento psico-motor nos casos 3 e 6, distúrbio de comportamento no caso 8 e hemiplegia no caso 10 .

Possiveis fatôres etiológicos puderam ser identificados em 14 pacientes, sendo os traumatismos de parto ( 8 casos) e as meningencefalites (4 casos) os mais frequientes (quadro 1). Nos 8 restantes, a história e o exame neurológico não foram suficientes para esclarecer a provável etiologia.

Em 15 casos $(68 \%)$ as descargas foram representadas por espiculas de elevado potencial, isoladas ou em grupos, com nitida reversão de fase no vértex (figs. 1, 2 e 3). Essas espiculas algumas vêzes apareceram bem localizadas na linha média e, em outras se propagaram às regiões rolândicas com predominio ou não em um dos hemisférios. De aparecimento espontâneo durante a vigília e pouco modificadas pela hiperpnéia, foram freqüentemente ativadas pelo sono.

Em 7 pacientes o foco parassagital estava representado principalmente por ondas lentas ( 1 a $4 \mathrm{c} / \mathrm{s}$ ) de elevado potencial, também com reversão de fase no vértex (casos 2, 4, 5, 13, 14, 17 e 22; figs. 4, 5, 6 e 7). Essas ondas apareceram por surtos nos casos $2,4,5 \in 22$ e de modo contínuo sôbre 0 vértex nos casos 3, 14 e 17. Deve-se ressaltar que 4 dêsses 7 pacientes (casos 4, 13, 14 e 22) apresentavam crises acinéticas (crises de perda de tonus) de dificil contrôle. Um dos pacientes (caso 14), ao lado de crises acinéticas e crises motoras localizadas no membro inferior esquerdo, desenvolveu um quadro hipotônico continuo, prolongado (3 meses de duração) e reversível. Esse paciente, durante o periodo de estado, apresentava disritmia paroxística permanente ao EEG, ảs vêzes com predomínio na região parassagital. Essa disritmia, presente sòmente durante o sono, dava lugar a descargas focais ao nivel do vértex durante a vigília e sonolência (figs. 2,3 e 7 ).

Surtos irregulares de complexos espícula-ondas difusas e bilaterais estiveram presentes em 6 pacientes $(\operatorname{casos} 3,12,13,14,15$ e 20 ; fig. 1$)$. Dêstes, apenas 2 (casos 3 e 20) apresentavam crises de ausência. Um terceiro paciente, também portador de crise de ausência (caso 5), apresentava ao EEG sòmente foco parassagital, devendo-se ressaltar, entretanto, que êsse traçado foi realizado apenas durante o sono. Além do distúrbio de consciência, êsses 3 pacientes apresentavam outras manifestações clínicas como versão dos olhos e da cabeça, sintomas vegetativos, incontinência urinária ou clonias no membro inferior.

Nos casos 18 e 19 apareceram espicula-ondas difusas e bilaterais, bastante trequentes porém isoladas.

\section{COMENTARIOS}

Nossos resultados, embora analisados sob os pontos de vista clínico e eletrencefalográfico, sem confirmação cirúrgica, eletrocorticográfica ou anátomo-patológica, nos levam a admitir que os 22 pacientes estudados apresentavam lesões epileptógenas localizadas na superfície mesial de um dos hemisférios cerebrais. 


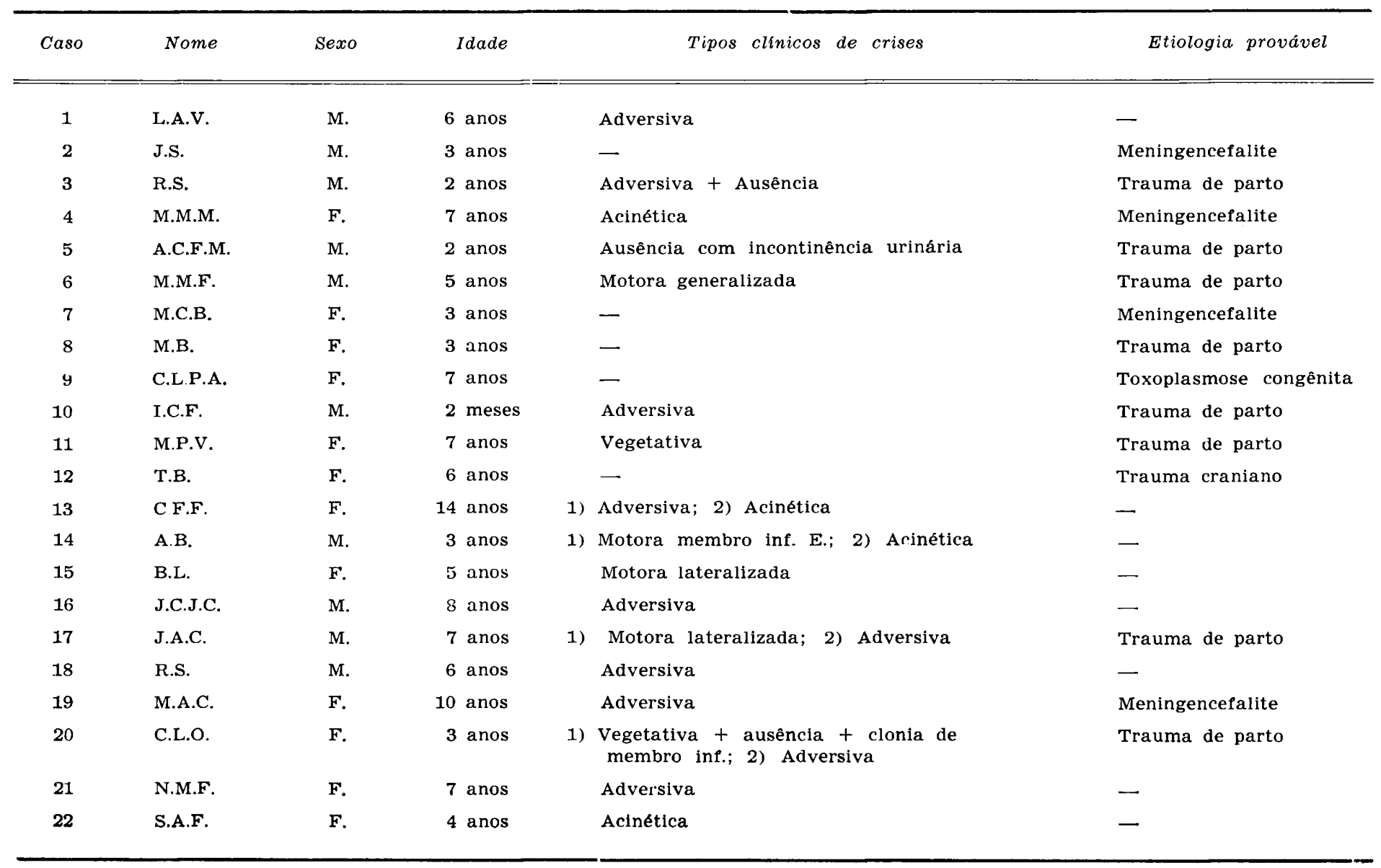

Quadro 1 - Dados de identificação, tipos clinicos de crises e provavel etiologia dos 22 casos estudados. 


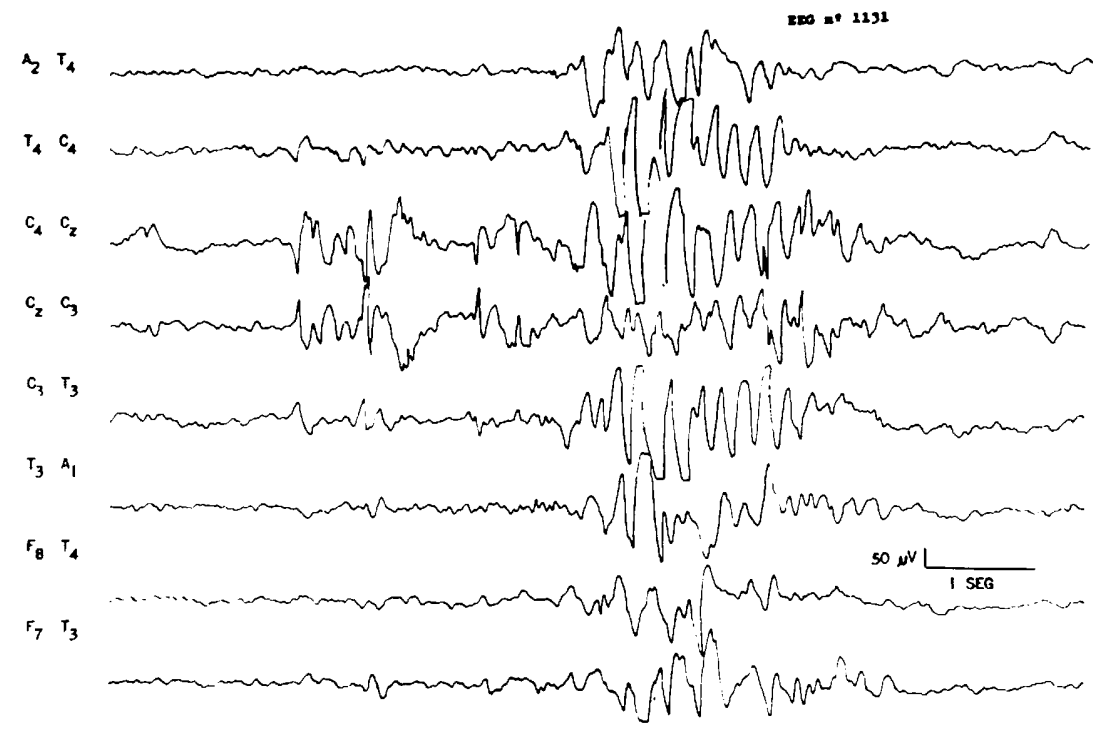

Fig. 1 - Caso 15. Traçado realizado em vigúlia. Espículas com reversão de fase ao nivel do vértex, seguidas de sincronismo bilateral secundário.

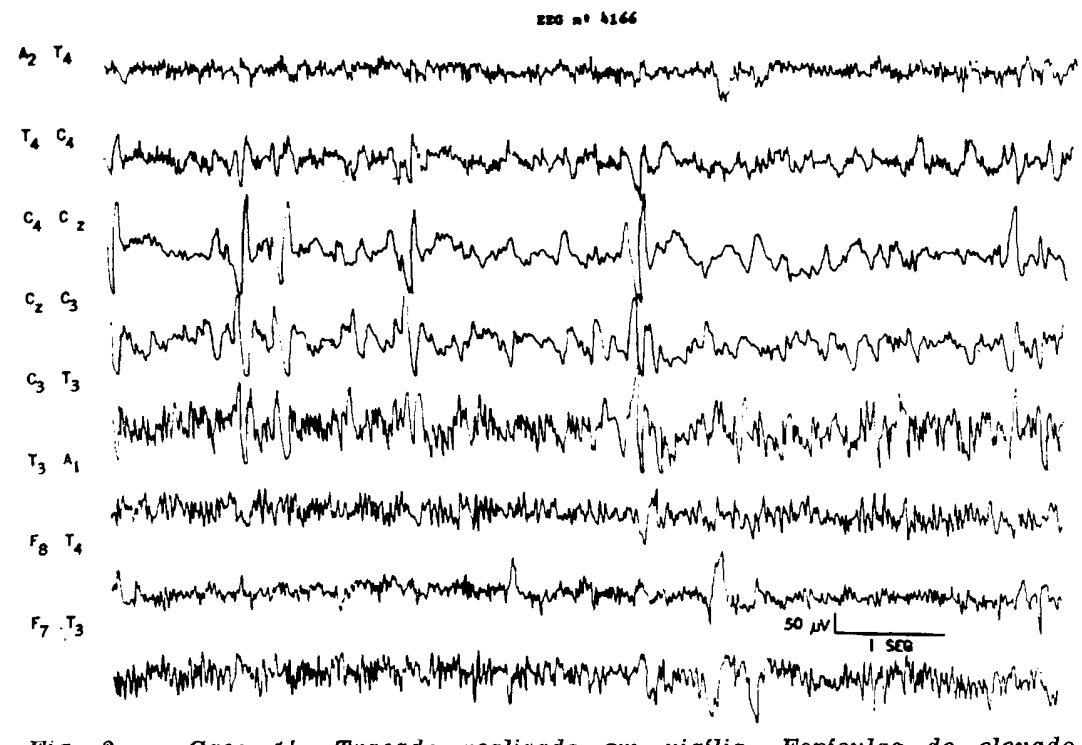

F'ig. 2 - Caso 14. Traçado realizado em vigilia. Espiculas de elevado potencial, isoladas, com reversão de fase no vértex. 


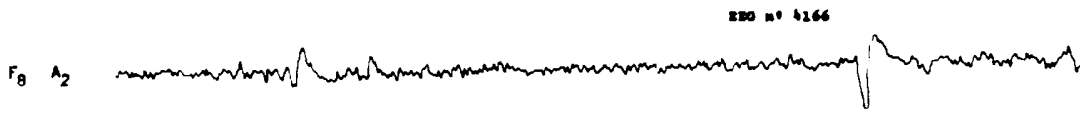

F7 A

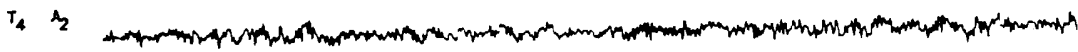

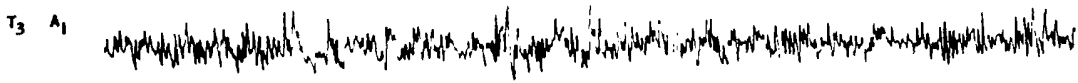

$T_{6} A_{2}$

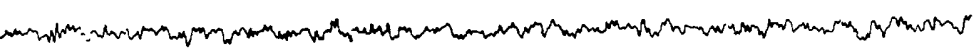

$T_{5}$ A

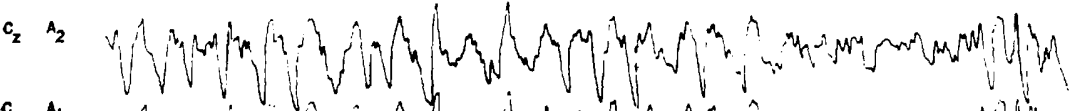

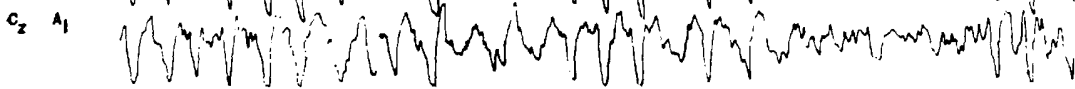

H'ig. 3 - Caso 14. Traçado realizado em vigilia. Espiculas ritmadas de projeção ao nivel do vértex.

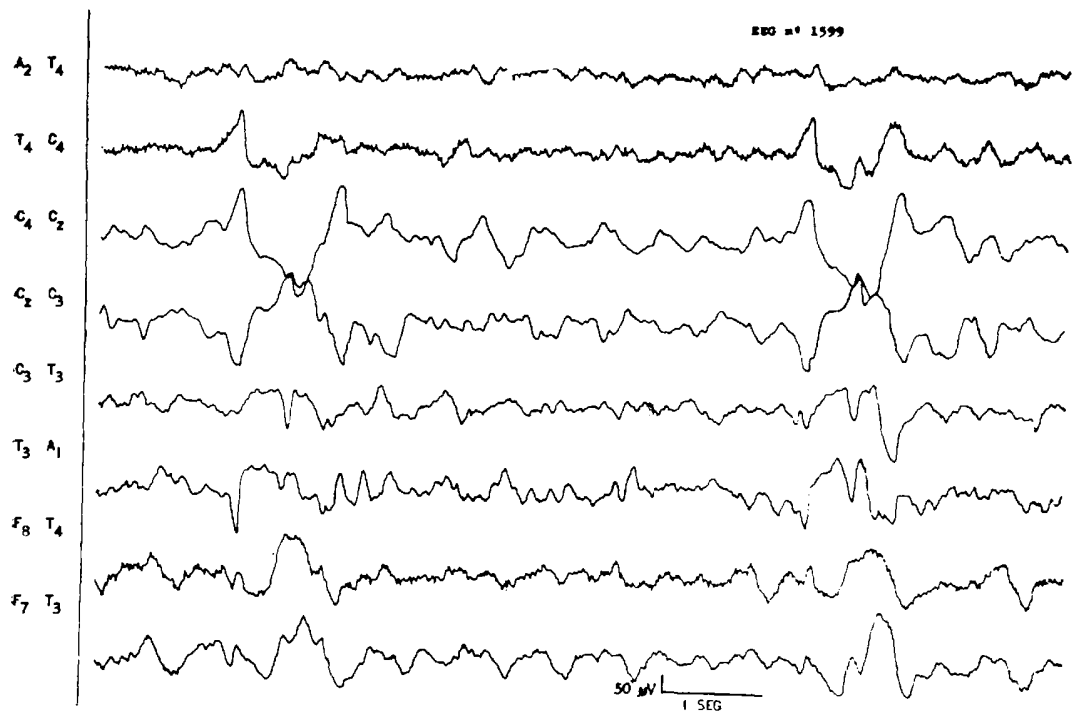

Fig. 4 - Caso 4. Tracado realizado em vigília. Ondas lentas irregulares, de $1 \mathrm{c} / \mathrm{seg}$ com reversão de fase no vértex. 
$2 T_{4}$

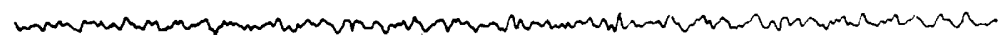

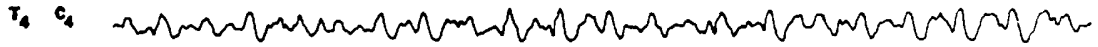

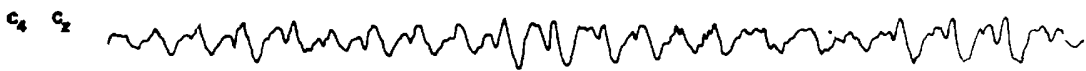

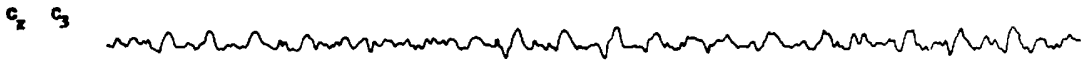

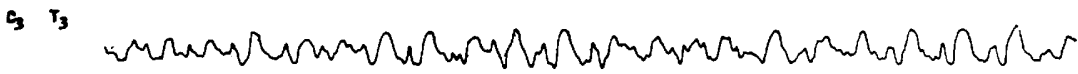

T3 1

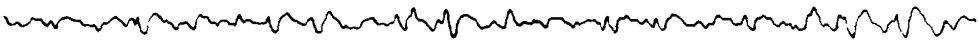

$F_{n} T_{4}$

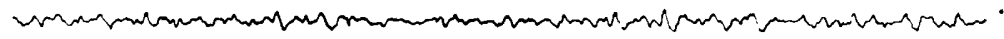

$F_{7} T_{3}$

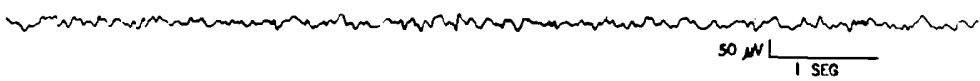

Fig. 5 - Caso 19. Traçado realizado em vigilia. Ondas lentas irregulares. de $3-4 \mathrm{c} / \mathrm{seg}$ com reversão de fase no vértex.

$=00000$

F. 3 2010

$\boldsymbol{F}_{\mathbf{1}} \mathbf{A}_{1}$

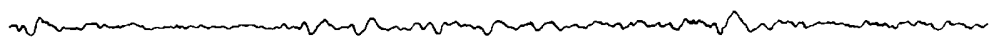

$T_{4} r_{2}$

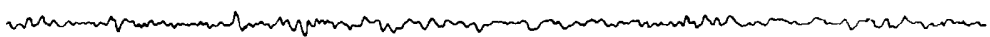

$T_{3} A_{1}$ ceron

$T_{6}{ }_{2}$

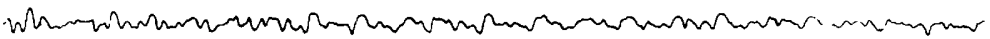
$T_{5} A_{1}$ so $\mathrm{NVL}$ I see

$c_{z} A_{2}$

$c_{2} A_{1}$

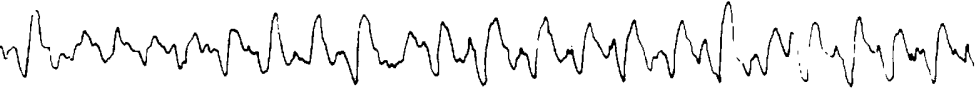

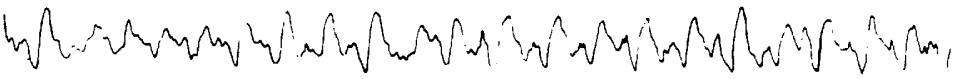

Fig. 6 - Caso 19. Traçado realizado em vigilia. Ondas ritmadas de $\$-4 \mathrm{c} / \mathrm{seg}$ de projeção ao nivel do vértex. 


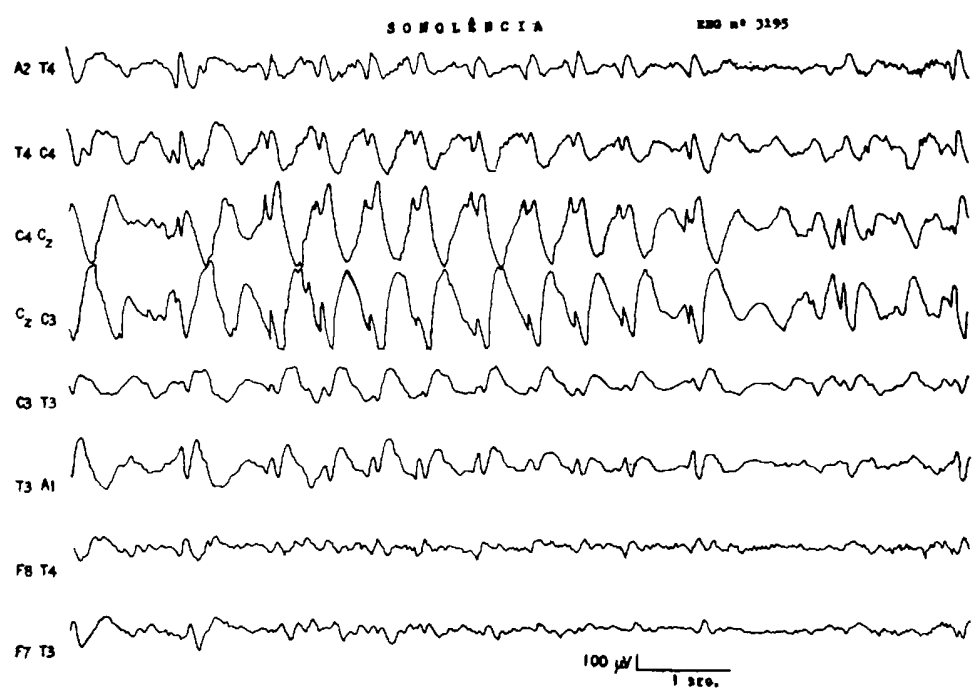

Fig $\gamma$ - Caso 14. Tracado realizado no decorrer de sonolência. Presença de espicula-ondas lentas com reversão de fase do componente lento ao nivel do vértex.

Dos 5.000 pacientes por nós estudados, apenas $22(0,5 \%)$ apresentavam foco convulsiógeno parassagital. Incidência de mesma ordem foi encontrada por Gastaut 5. Deve-se salientar, entretanto, que êsses resultados não exprimem a verdadeira incidência das lesões epileptógenas situadas na superfície mesial do hemisfério cerebral, desde que descargas focais primárias ali criginadas dificilmente são registradas através de eletrodos extracranianos $17,19$.

Com relação à idade, houve nítido predomínio na infância. Dentre os pacientes estudados por Bancaud e col. ${ }^{2}$ a idade máxima foi de $131 / 2$ anos, com uma incidência predominante entre os 3 e 6 anos. A idade média dos pacientes de Tükel e Jasper ${ }^{19}$ e Kennedy ${ }^{9}$ situa-se bem acima daquela por nós encontrada.Devemos salientar, entretanto, que êsses autores empregaram ativação pelo Metrazol ou Megimide em alguns de seus traçados, o que não ocorreu em relação ao nosso material.

A maior incidência de foco parassagital em crianças estaria relacionada com o progressso de maturação cerebral ${ }^{2,8}$. Com o desenvolvimento dêsse processo haveria aumento do limiar convulsiógeno daquela região cerebral. Isto nos leva a admitir a necessidade de processos de ativação (Metrazol, Megimide, etc.) ao se obter o EEG de pacientes, principalmente adultos, com suspeita de patologia na superficie interhemisférica.

Penfield e Jasper ${ }^{15}$ e Jasper ${ }^{6}$ relataram o aparecimento de descargas ritmicas sincronas e bilaterais em pacientes com foco epileptógeno na superficie mesial de um dos hemisférios. Achados semelhantes foram descritos por Tükel e Jasper ${ }^{19}$ e Marossero e col. ${ }^{13}$. Por outro lado, Kennedy ${ }^{9}$ não encontrou qualquer evidência de sincronismo bilateral em 7 pacientes por 
êle estudados. Entretanto, a julgar pelo diagrama dêsse autor, em apenas um caso a lesão situava-se na superfície mesial, sendo que nos 6 restantes estava localizada na borda superior do hemisfério. Aquelas descargas foram denominadas "sincronismo bilateral secundário" 19 (SBS) para diferenciá-las do sincronismo bilateral primário (SBP) da epilepsia Pequeno Mal clássico que, como se sabe, tem origem em estruturas subcorticais.

De acôrdo com Penfield e Jasper ${ }^{17}$, o SBS estaria relacionado com lesões situadas na área motora suplementar ou no córtex que circunda o "genu" oli o esplênio do corpo caloso. Ralston ${ }^{18}$, em estudos experimentais em gatos, encontrou evidências de que levões epilptógenas situadas no giro cingulado são capazes de induzir descargas sincronas difusas e bilaterais. Essas descargas seriam mediadas através de centros subcortais, provàvelmènte pela formação reticular talâmica medial.

A diferenciação entre o SBS e SBP, embora difícil em alguns casos, pode ser estabelecida com base nos achados descritos por Tükel e Jasper ${ }^{19}$. Êsses autores encontraram alguns elementos eletrográficos que caracterizam SBS: 1) irregularidade da descarga; 2) reverssão simples de fase na linha média; 3) área equipotencial sôbre a região lesada, sendo de baixa voltagem em lesőes destrutivas; 4) durante crise cliníca, as descargas em áreas contralaterais ao foco, apresentam maior voltagem; 5) supressão da atividade elétrica precedendo ou acompanhando crise clínica.

Em nosso material 6 pacientes apresentavam SBS em seus traçados (fig. 1). Êsse sincronismo caracterizou-se fundamentalmente por 3 elementos: 1) irregularidade das descargas; 2) reversão simples de fase na linha média; 3) assimetria de voltagem em tôrno do vértex.

Do ponto de vista clínico o SBS esteve associado a crisses de ausência em apenas dois de nossos pacientes. Entretanto, essas crises assumiram características diferentes do Pequeno Mal clássico de origem "centrencefálica" pois, ao lado do distúrbio de consciência, eram acompanhadas de outras manifestações, como versão dos olhos e da cabeça, sintomas vegetativos, incontinência urinária ou clonias no membro inferior. $O$ mesmo ocorreu em relação aos casos descritos por Tükel e Jasper ${ }^{19}$ e Mazars e col. ${ }^{14}$.

A origem focal dessas crises deve, portanto, ser levada em consideração, principalmente se associadas a descargas com caracteristicas de SBS.

Em nosso material houve predominio das crises adversivas, presentes em $45 \%$ dos casos. A área motora suplementar e o córtex frontal anterior interhemisférico são as regiões mesiais mais estritamente relacionadas com êsse tipo de crise $1,16,17,19$.

A ocorrência de crises acinéticas em 4 pacientes com foco epileptógeno parassagital nos chamou particularmente a atenção. Em todos êles o foco esteve representado por ondas lentas com reversão de fase no vértex. Achado semelhante foi descrito em um caso de Courjon e Favel ${ }^{4}$.

Mazars e col. ${ }^{14}$ estudando manifestações clínicas das epilepsias "cinguladas" encontraram modificaçōes tônicas do segmento cefálico em 10 dos 20 pacientes que apresentavam crises de ausência. 
De acôrdo com Livingston ${ }^{12}$, Chao e col. ${ }^{3}$ e Lennox ${ }^{11}$, as crises acinéticas estão freqüentemente associadas a lesões orgânicas cerebrais, congênitas ou adquiridas, sendo portanto a expressão de uma epilepsia sintomática. Por outro lado, há evidências de que a estimulação do giro cingulado em animais pode determinar perda súbita de tono muscular, semelhante àquela verificada em pacientes com crise acinética ${ }^{10}$.

Essas observações sugerem a participação do córtex mesial na fisiopatogenia das crises acinéticas.

\section{RESUMO E CONCLUSOES}

Foram estudados, do ponto de vista clínico e eletrencefalográfico, 22 pacientes que apresentavam foco convulsiógeno parassagital com reversão de fase no vértex. O critério adotado na seleção dos casos foi puramente eletrencefalográfico. Dêsse estudo os autores concluem:

1. Descargas focais primárias ao nível da região parassagital são raras $(0,5 \%$ aproximadamente) na ausência de processo medicamentoso de ativação; predominam nitidamente na infância.

2. Através de centros subcorticais (provàvelmente a formação reticular talâmica medial), podem determinar o aparecimento de descargas síncronas difusas e bilaterais ("sincronismo bilateral secundário"), que, em nosso material, esteve presente em 6 casos $(27 \%)$.

3. Estas descargas, às vêzes semelhantes ao sincronismo bilateral primário da Epilepsia Pequeno Mal clássica, caracterizaram-se por 3 elementos fundamentais: a) irregularidade das descargas; b) reversão simples de fase na linha média; c) assimetria de voltagem em tôrno do vértex.

4. Do ponto de vista clínico houve predominio das crises adversivas $(45 \%$ ) e das crises acinéticas (18\%) sôbre as demais.

5. Sincronismo bilateral secundário pode $s \in \mathrm{r}$ acompanhado de crises de ausência (em nosso material presente em apenas 2 casos) que diferem daquelas típicamente "centrencefálicas" pela variabilidade de manifestações que as acompanham.

6. Lesões epiletógenas localizadas na superfície mesial de um dos hemisférios cerebrais poderiam ser responsabilizadas pelo aparecimento de crises acinéticas. Essas crises estiveram presentes em 4 casos e, em todos êles, a descarga focal esteve representada por ondas lentas (1 a $4 \mathrm{c} / \mathrm{s}$ ), de elevado potencial e com reversão de fase no vértex.

\section{SUMMARY AND CONCLUSIONS}

Parasagittal epileptogenic focus with a phase reversal at the vertex: clinical and electroencephalographic correlations

The authors studied, from the clinical and electroencephalographic points of view, 22 patients with parasagittal convulsive focal activity with a phase 
reversal at the vertex. The selection of cases was purely electrocncephalographic. The authors conclude:

1. Primary focal discharges in the parasagittal region are rare $(0,5 \%)$ when the EEG is not activated; they are more frequently found in infancy and childhood.

2. The parasagittal focus may lead to bilateral diffuse synchronous discharges (secondary bilateral synchronism) through some subcortical centers (presumably the thalamic reticular formation). Those discharges were present in 6 cases.

3. Those discharges can be similar to the primary bilateral synchronism found in the P.M. epilepsy and can be characterized by three fundamental aspects: a) irregularity of discharges; b) a single phase reversal at the midline; c) asymmetry at the vertex region.

4.) From the clinical point of view the adversive (45\%) and akinetic seizures $(18 \%)$ were the most frequently found.

5. Secondary bilateral synchronism can be associated with absence seizures (observed in 2 cases only); these seizures however are very different from thcse called "centrencephalic" for they are associated with several types of clinical. signs.

6. Epileptogenic lesion in the mesial aspect of cerebral cortex could be responsible for akinetic seizures. The authors found these seizures in 4 patients and in all of them the focal discharge was represented by slow waves ( 1 to $4 \mathrm{c} / \mathrm{sec}$ ) of high voltage and with a phase reversal at the vertex.

\section{REFERENCIAS}

1. AJMONE-MARSAN, C. \& RALSTON, B. L. - The Epileptic Seizure. Its: Functional Morphology and Diagnostic Significance. Charles C. Thomas,. Spring field (Illinois), 1957.

2. BANCAUD, J.; COLOMB, H. \& DELL, M. B. - Rolandic spikes: an EEG sign typical for childhood. EEG Clin. Neurophysiol. 10:346, 1958.

3. CHAO, D.; DRUCKMAN, R. \& KELlAWAY, P. - Convulsive Disorders of Children. W. B. Saunders Co., Philadelphia-Montreal, 1958.

4. COURJON, J. \& FAVEL, P. - L'aspect electrographique des crises akinétiques. Rev, Neurol. 105:211-212, 1961.

5. GAS'lAUT, Y. - Les pointes negatives évoquées sur le vertex. Leur signification psychophysiologique et pathologique. Rev. Neurol. 89:382-399, 1953.

6. JASPER, H. - Etude anatomo-physiologique des epilepsies. EEG Clin. Neurophysiol. Suppl. 2:99-111, 1951.

7. JASPER, H. - The ten twenty system of the international federation. EEG clin. Neurophysiol. 10:371-375, 1958. 
8. KELlawAY, P. \& PETERSEN, I. - Neurological and Electroencephalographic Correlative Studies in Infancy. Grune \& Stratton, New York-London, 1960.

9. KENNEDY, W. A. - Clinical and electroencephalographic aspects of epileptogenic lesions of the mesial surface and superior border of the cerebral hemisphere. Brain 82:147-161, 1959.

10. LENNOX, M. A. \& ROBINSON, F. - Cingulate cerebellar mechanisms in the physiological pathogenesis of epilepsy. EEG Clin. Neurophysiol. 3:197-205, 1951.

11. LENNOX, W. G. - Epilepsy and Related Disorders. Little Brown Co., Boston, 1960.

12. LIVINGSTON, $\mathrm{S}$. - The Diagnosis and Treatment of Convulsive Disorders in Children. Charles C. Thomas, Springfield (Illinois), 1954.

13. MARUSSERO, F.; MASPES, P. E. \& RIVOLTA, A. - Post-traumatic focal epilepsy with parasagital focus; clinical, electroencephalographic, electrocarticographic observations. EEG Clin. Neurophysiol. 6:533, 1954.

14. MAZARS, Y.; MAZARS, G.; GOTUSSO, C. \& MERIENNE, L. - Place de l'épilepsie cingulaire dans le cadre des épilepsies focales corticales. Rev. Neurol. 114:225-24i, 1966.

15. PENFIELD, W. \& JASPER, H. - Highest level seizures. Proc. Res. Publ. Ass. Nerv. Ment. Dis. 26:252-271, 1946.

16. PENFIELD, W. \& WELCH, K. - The supplementary motor area of the cerebral cortex. A. M. A. Arch. Neurol. \& Psychiat. 66:289-317, 1951.

1\%. PENFIELD, W. \& JASPER, H. - Epilepsy and the Functional Anatomy of the Human Brain. Little Brown Co., Boston, 1954.

18. RALSTON, B. L. - Cingulate epilepsy and secondary bilateral synchrony. EEG Clin. Neurophysiol. 13:591-598, 1961.

19. TUKEL, K. \& JASPER, H. - The electroencephalogram is parasagital lesions. ÉEG Clin. Neurophysiol. 4:481-494, 1952.

Departamento de Neurologia - Faculdade de Medicina - Caixa Postal 301 Kibeırào Prêto, SP. - Brasil. 